\title{
VARIANTEN VON VIRTUOSITÄT. INNOVATIONEN DES JAZZAVANTGARDISTEN ANTHONY BRAXTON
}

\author{
Timo Hoyer
}

\section{Verständnis von Virtuosität}

Wer lateinisch geschulte Ohren hat (oder einfach bei Wikipedia nachschaut), hört im Wort Virtuosität den Ausdruck virtus, der gewöhnlich mit Tugend übersetzt und mit moralischen Charaktereigenschaften in Verbindung gebracht wird. Nach der ursprünglichen Bedeutung sind mit virtus allerdings Wesensmerkmale der Männlichkeit (vir) gemeint. Etwas später erhielt der Ausdruck in der römischen Antike die Bedeutung von vorzüglichen Attributen, die meistens, aber nicht ausschließlich, ethischer Natur waren (vgl. Hoyer 2005: 79f.). Der Ausdruck Virtuosität hat etwas von dieser Bedeutung bewahrt, wenngleich er nicht auf virtus oder Tugend zu reduzieren ist.

Virtuosität symbolisiert eine Form praktischer Vorzüglichkeit, die das Gegenteil von Unfähigkeit und Dilettantismus ist. Deshalb kann in einem Beitrag über das zeitgenössische Musiktheater die Bemerkung fallen: »Nie war der Grat zwischen Virtuosität und Dilettantismus so schmal« (LemkeMatwey 2016: 49), was impliziert, besagter Grat sei üblicherweise breit. Virtuosität ist - wie virtus - zumeist ein werthaltiger Begriff, der eine domänenspezifische, hervorragende Könnerschaft bezeichnet, was überwiegend positiv besetzt ist. Ein Virtuose beherrscht sein Metier gut, auffällig gut. In diesem Sinne lässt der Liedermacher und Dichter Jan Böttcher in seinem Roman Das Lied vom Tun und Lassen seine Hauptfigur, einem in die Jahre gekommenen Musiklehrer, selbstkritisch feststellen, er sei höchstens ein brauchbarer Rhythmusgitarrist, aber: „Kein Solist und schon gar kein Virtuose (Böttcher 2011: 72).

Virtuosität erschöpft sich nicht in der meisterhaften Erfüllung anspruchsvoller Erwartungen und Normen; das wäre bei Böttcher die Rubrik des Solisten. Um als Virtuose zu gelten, müssen Momente der Übererfüllung 
von Aufgaben, des Exorbitanten, der verblüffenden Normabweichung, der situativen Steigerung hinzukommen. Noch ein Beispiel aus der Belletristik: Gabriel García Márquez schreibt über eine Person, die sich in die Planung eines Begräbnisses hineinsteigert, sie sei »mehr als eine Spezialistin«, nämlich »eine Virtuosin in Sachen Tod« (Márquez 1997: 315).

Nicht zufällig bestand das Motto der Tagung zum Thema Virtuosität, auf der die Urfassung dieses Beitrags zur Diskussion gestellt wurde, aus Komparativen: höher, schneller, lauter - als ginge es um Leistungssport. Tatsächlich hat Virtuosität einen sportlichen Charakter. Wie eine Sportart kann Virtuosität bis zu einem gewissen Grad trainiert, geübt werden. Und wie beim Leistungssport nimmt Virtuosität Maß an anderen, vergleichbaren Leistungen. Wenn es eine Virtuosin in Sachen Tod gibt, dann muss es zumindest vorstellbar sein, dass es Menschen gibt, die die Sache ähnlich, weniger oder gar nicht virtuos betreiben. In der Musik werden solche Maßstäbe meistens mit bestimmten Personen verbunden: Ein Bebop-Saxophonist etwa muss sich als Virtuose an Charlie Parker messen, ein Cool Jazz-Saxophonist an Lee Konitz, ein Bluesrock-Gitarrist, je nach Stil, an Buddy Guy, Eric Clapton, Johnny Winter oder Stevie Ray Vaughan, ein Heavy Metal-Sänger, je nachdem, an Robert Plant oder Attila Csihar.

Virtuosität folgt, auch darin dem Sport verwandt, einer Steigerungs- und Bestmarkenlogik. Was ehemals als virtuos galt, kann deshalb mit der Zeit wie gehobener Dilettantismus wirken (man schaue sich nur alte Fernsehaufzeichnungen von Olympischen Spielen oder Fußballweltmeisterschaften an). Lightnin' Hopkins hat seinen Spitznamen einst erhalten, weil man ihn für sein blitzschnelles Gitarrenspiel bewunderte. Heute würde man ihn eher Slowhand Hopkins taufen, wobei auch Langsamkeit - siehe Clapton, siehe Konitz - in Abhängigkeit vom musikalischen Beurteilungsmaßstab eine Virtuositätsnorm abgeben kann.

Manche Instrumentalisten wie Charlie Parker oder Jimi Hendrix erwerben sich den legendären Ruf, in puncto Virtuosität unübertreffbar zu sein, obgleich ihnen mittlerweile in technischer Hinsicht einige das Wasser reichen. Virtuosität ist folglich nicht unbedingt gleichbedeutend mit angelernter technischer Bravour, also nicht bloße Spitzenleistung. Das unterscheidet sie vom Leistungssport, wo am Ende allein das messbare Resultat zählt. Drängt sich die technische Meisterschaft penetrant in den Vordergrund, verwandelt sich Virtuosität sogar ins Zirkushaft-Artistische. Der Wertbegriff kann dann eine pejorative Bedeutung annehmen. Ein letztes Beispiel aus der Literatur: In Gottfried Kellers Der grüne Heinrich leidet der Protagonist darunter, dass er es zwar fertigbringe, dank seiner »eingelernten Pinselvirtuosität« (Keller 1919: 293) ansehnliche Bilder zu malen, diese seien aber 
ohne Gehalt und Seele, schnöde Kunstfertigkeit. Kraft seiner »lächerlichen Virtuosität« (ebd.: 298) überdeckt er lediglich seinen Mangel an Poesie. Jedem fallen, nehme ich an, auch aus der Musikbranche solche Fälle lächerlicher Virtuosität ein, wo künstlerische Substanz von technischer Brillanz überlagert wird.

Wobei zu fragen ist, ob es sich überhaupt um Virtuosität handelt, wenn Instrumentalisten hochkomplizierte, irrsinnig anspruchsvolle Leistungen wie Zirkusnummern oder eingelernte Kabinettstücke abrufen. Dann wäre ja das Player Piano von Conlon Nancarrow unschlagbar virtuos. Doch das Adjektiv will einem hier nicht über die Lippen. Und warum nicht? Die unmenschlich schnellen Tonkaskaden lassen keinen Zweifel, dass dort ein programmiertes Gerät am Werk ist. Die Mechanik ist fremdbestimmt, sie verfügt über keine Gestaltungs- und Ausdrucksfreiheit, sie kennt nicht die Möglichkeit des Scheiterns, sie funktioniert oder ist defekt. Das ist unvereinbar mit dem Begriff von musikalischer Virtuosität, der auf nichtdeterminierte künstlerische Handlungen rekurriert und Fehlbarkeit einschließt. Buchstäblich gestanzte, mechanische Virtuosität ist deshalb nicht einmal lächerlich, sondern überhaupt keine Virtuosität.

Ich halte die geläufige Unterscheidung von klassischer europäischer Kunstmusik (E-Musik) und populärer Musik (U-Musik) für unsinnig und hinfällig (vgl. Hoyer 2017). Aber so verkehrt und unscharf die Trennung ist, so unscharf und oberflächlich möchte ich behaupten, die »populäre Musik « hat das Erscheinungsbild und den Stellenwert von Virtuosität immer dort am stärksten geprägt, wo sie den Performancekünstlerinnen und -künstlern umfangreiche Gestaltungsspielräume gewährte. Damit sind wir beim Jazz und bei der Improvisation angelangt.

\section{Virtuosität im Jazz}

Zu diesem Punkt nur kurz und thesenhaft: Der Jazz hat mit dem für diese Musikrichtung elementaren »Prinzip Freiheit« (John Litweiler) zugleich das Element der Virtuosität wie kaum eine andere Musikform kultiviert. Man kann darüber streiten, ob Virtuosität im Jazz das Salz in der Suppe oder gar die Suppe selbst ist, unstrittig dürfte sein, dass im Jazz Gestaltungsfreiheit und Virtuosität substantielle Faktoren sind.

Der tieferliegende Grund dafür ist in dem Umstand zu suchen, dass der »Lebensnerv des Jazz« (Steve Lacy) auf Improvisation beruht (vgl. Feige 2014). Im traditionellen Jazz - ebenso wie in allen anderen Musikformen, 
die umfangreiche Improvisationsanteile enthalten (vgl. Bailey 1987) - ist Virtuosität vorrangig eine Domäne des improvisierenden Musikers.

Die Jazz-Avantgarde hat seit den 1960er Jahren die Parameter des Jazz von Grund auf reformuliert und dekonstruiert. Die erste und zweite US-amerikanische Generation der Avantgardisten - Ornette Coleman, John Coltrane, Sun Ra, Cecil Taylor, Albert Ayler, Archie Shepp etc. (vgl. Litweiler 1988; Wilmer 1992) - und die frühen europäischen Improv- und Free Jazz-Musiker - Derek Bailey, Peter Brötzmann, Evan Parker, Han Bennink etc. (vgl. Noglik 1981) - revolutionierten und erweiterten vor allem das Improvisationsspektrum und stärkten somit die Stellung des Virtuosen.

Zu fundamentalen Neudefinitionen von Improvisation und Virtuosität kam es dann vor allem in der dritten US-amerikanischen Generation der Jazzavantgarde. Vorneweg die Mitglieder der »Association for the Advancement of Creative Musicians « (AACM) erarbeiteten umwälzende musikalische Konzepte, die nicht selten den Rahmen des Jazz sprengten (vgl. Lewis 2008). Davon handeln die anschließenden Überlegungen, die einen Musiker dieser Organisation ins Zentrum rücken: Anthony Braxton.

\section{Vier Varianten von Virtuosität}

Anthony Braxton, 1945 in Chicago geboren, zählt neben Muhal Richard Abrams, George Lewis, Roscoe Mitchell, Leo Smith und Henry Threadgill bis auf den Tag zu den produktivsten Multiinstrumentalisten, Komponisten und Theoretikern aus den Reihen der AACM. Das Konzept der Virtuosität wird von Braxton zugleich modifiziert und unterlaufen. Seine Herangehensweise zeichnet Wege vor, die der progressive Jazz seit den 1970er Jahren einschlagen sollte. Es muss freilich anderen Arbeiten vorbehalten bleiben, seinen vielfach bezeugten Einfluss auf die nachfolgenden Generationen von, sagen wir, John Zorn, Ken Vandermark, Angelika Niescier und Mary Halvorson unter den hier thematisierten Gesichtspunkten aufzuzeigen.

Wenn im Folgenden rekonstruiert wird, wie das Phänomen der Virtuosität im Werk Braxtons in Erscheinung tritt, dann geschieht dies, ohne dass er selbst an irgendeiner bedeutsamen Stelle seiner breit angelegten Musikästhetik (Braxton 1985) oder in einem seiner ungezählten Interviews das Thema der Virtuosität von sich aus angeschnitten hätte. Gleichwohl kann der analytische Zugriff in seinem gewaltigen CEuvre vier Varianten von Virtuosität ausfindig machen. 


\subsection{Selbstbestimmte Virtuosität}

Insbesondere in der ersten Hälfte seiner musikalischen Laufbahn - sie umfasst nach meiner Einschätzung den Zeitraum 1967 bis 1993 - treibt Braxton in einer bestimmten Hinsicht das virtuose Spiel auf seinen einheimischen Instrumenten (Saxophon und Klarinette) ins Extrem. Das geschieht vorneweg in seiner Solomusik für Altsaxophon (vgl. Heffley 1996: 236ff.; Lock 1995). Bis zur Veröffentlichung seines Doppelalbums For Alto war, von punktuellen Ausnahmen abgesehen, das unbegleitete Spiel im Jazz eine Domäne von Pianisten und Gitarristen. Als der noch weitgehend unbekannte 23-jährige sich 1968/69 erkühnte, gleich vier Plattenseiten mit nichts anderem als seinem Altsaxophon einzuspielen - lauter Eigenkompositionen -, war das ein Wagnis, das Türen öffnete. Seitdem ist der unbegleitete Auftritt im Jazz jedem Instrument, auch der Stimme, erlaubt; Soloaufnahmen gibt es mittlerweile in Hülle und Fülle, wenngleich überwiegend von eher experimentellen Künstlerinnen und Künstlern.

Braxton ließ seiner Pioniertat in kurzen Abständen weitere Soloeinspielungen und zahllose Solokonzerte folgen. Neben Anerkennung brachte ihm das unter Kollegen und von Kritikerseite auch den Vorwurf des Egozentrismus ein: Hier pfeife einer auf das kollektive Miteinander, was als Regelbruch diskreditiert wurde. Phil Woods etwa hat sich derart geäußert (Radano 1993: 181). Ähnliche Maßregelungen hatte bereits die erste Generation der Jazzavantgarde zu hören bekommen, damals allerdings waren die Kollektivimprovisationen Stein des Anstoßes (vgl. Jost 1982: 181). Als moralisches Urteil ist der Egozentrismus-Vorwurf, man kann es nicht anders sagen, dämlich. Doch ein markanter Regelbruch lag bei Braxton in der Tat vor. Nach konventionellem Verständnis benötigt der Jazzvirtuose, jedenfalls wenn er ein single line-Instrument spielt, einen ihn einbettenden Rahmen, ein containment. Dieses containment bilden herkömmlicherweise die Mitspieler, aber auch bekannte Performancestrukturen (z.B. Head-Solo-Head) und harmonische, melodische und rhythmische Grundmuster. Der klassische Jazz besteht im Allgemeinen aus der Kombination dieser drei Elemente. Innerhalb dieses Rahmens dürfen sich die Instrumentalisten, ohne dass ein Phil Woods die Egozentrismus-Keule schwingen würde, solange solistisch verausgaben, wie es ihnen beliebt. Der gezielte Regelbruch des Solomusikers Braxton bestand darin, dass er auf diese Rahmenbedingungen komplett verzichtete. Damit fügte er dem Jazz eine neue Gestalt hinzu, für viele Liebhaber der Jazzmusik eine durchaus inakzeptable. 
Mit dem Verzicht auf das übliche containment ist der Solist in jeder Hinsicht auf sich allein gestellt. Der Instrumentalist muss sich und sein Spiel im wahren Sinne des Wortes selbstbestimmen, er ist, mit einem anderen Ausdruck, autark. Auch als Virtuose. Mit der Abwendung vom geläufigen Jazzrahmen verlieren die darin implementierten Qualitäts- und Virtuositätsmaßstäbe mit einem Schlag an Geltung und Verbindlichkeit. Wer diesen gewagten Schritt ins musikalische Neuland partout abwerten will, soll ihn als egozentrisch oder ultra-individualistisch verhöhnen, doch damit verfehlt man das strukturell Neue daran.

Apropos Abwertung: Erfüllt Braxton überhaupt die Mindestvoraussetzung eines Saxophonvirtuosen: Ist er ein Meister seines Instruments? Das ist allen Ernstes in Zweifel gezogen worden. Phil Woods und andere lästerten über den Solomusiker und sprachen ihm das technische Vermögen ab (während er gleichzeitig in Down Beat in manchen Jahren zum besten Altsaxophonisten gekürt wurde). Auch solche Herabsetzungen mussten sich vor und neben Braxton andere Jazzavantgardisten gefallen lassen, die törichterweise vom bornierten Jazzestablishment als handwerklich inkompetent verspottet wurden (vgl. Jost 1982: 182). Wenn der Saxophonist Braxton kein Virtuose ist, dann habe ich keine Ahnung, was der Ausdruck bedeutet. Braxtons notengebundenes Spiel und seine Improvisationen sind sagenhaft kompliziert, anspruchsvoll, enorm abwechslungs- und einfallsreich, unverwechselbar und innovativ, und das in technischer, klanglicher wie struktureller Hinsicht. Virtuosität par excellence!

Ich lasse hier die einzelnen spieltechnischen Neuerungen Braxtons beiseite (über For Alto vgl. z.B. Radano 1993: 134f.). An dieser Stelle muss der Hinweis genügen, dass Braxton das musikalische Vokabular und das Klangspektrum für Holzblasinstrumente kontrolliert erweiterte. Nach der Verabschiedung der gängigen containment-Elemente ging er im Nu dazu über, ein eigenes Bezugssystem zu entwickeln: die Language Music. Diese setzt sich aus zwölf Kategorien zusammen: Nr. 1 Long Tones, Nr. 2 Accented Long Tones, Nr. 3 Trills, Nr. 4 Staccato Formings, Nr. 5 Intervallic Formings, Nr. 6 Multiphonics, Nr. 7 Short Attacks, Nr. 8 Angular Attacks, Nr. 9 Legato Formings, Nr. 10 Diatonic Formings, Nr. 11 Gradient Formings, Nr. 12 Sub-identity Formings (vgl. Wilson 1993: 98ff.; Wooley 2016b). Mit diesem zwölfteiligen Schema im Kopf organisiert Braxton sein kompositorisches Gesamtwerk, sein eigenes Instrumentalspiel und mitunter auch das der Mitspieler/innen. Wenn alles, was er an Musik bislang hervorgebracht hat, ein einziger komplexer Organismus ist, dann ist die Language Music, wie Braxton selbst manchmal sagt, dessen DNA. Mit der Language Music hat seine Musik ein selbstgeschaffenes containment erhalten. 
Im Rahmen dieses containments ist auch Virtuosität neu zu verorten. Sie ist erkennbar am kompetenten (verständnisvollen, vorzüglichen...) und kreativen (originellen, erfindungsreichen...) Umgang mit den Vorgaben des zwölfteiligen Vokabulars. Und mir scheint, als besäßen wir damit eine ganz brauchbare (nein, nicht erschöpfende) Definition von Virtuosität im Allgemeinen: kompetent-kreativer Umgang mit den Vorgaben eines bestimmten Vokabulars. Wenn ich im Weiteren von Virtuosität spreche, dann in diesem Verständnis.

\subsection{Untergrabene Virtuosität}

Die Frage ist noch offen, wie es angehen kann, dass Leute wie Phil Woods Braxton die vorhandene Spieltechnik absprechen? Es ist ähnlich wie beim Egozentrismus-Verdikt. Das respektlose Urteil ist zwar abwegig, aber die darin zum Ausdruck kommende Verunsicherung hat ihren nachvollziehbaren Grund. Braxton unterläuft als Instrumentalist und Komponist nicht nur die konventionellen Virtuositätsstandards, indem er das gewohnte Jazzcontainment hinter sich lässt. Obendrein untergräbt er mit Absicht Virtuositätsmaßstäbe, die auf Perfektion und Korrektheit ausgerichtet sind. Wie macht er das?

Um zunächst mit den Solokompositionen fortzufahren: Deren »Partituren«, wenn man sie so nennen mag, bestehen aus untereinander aufgereihten schriftlichen Spielanweisungen, gelegentlich ergänzt um Zeichen, bewegte Linien und Muster, aber nur höchst sporadisch aus Noten oder irgendwelchen Angaben, von denen man sagen könnte, wie sie exakt klingen müssten. Die Composition No. $106 a$ etwa enthält dreizehn Punkte, von denen die ersten acht hier genügen mögen:

1. Quarter Note Piece

2. Long Winding Sound Lines

3. Sound Line >Dips $<$ in the Middle

4. Long Sound >Winnie<

5. Long Sound >Curk $<$ - at the end of P.G.

6. >Tightish< Vibrato

7. >Bent< Sound Line Constructions

8. Curve Sound Line Shapes« (Braxton 1988: 259).

Der Interpret Braxton nimmt es bei der Realisation seiner eigenen Angaben nicht besonders genau, sie dienen ihm als Stimulus und ungefähre Orientierung, werden aber selten konsequent oder vollständig ausgeführt. In seinen Solokonzerten spielt er alle Stücke frei aus der Erinnerung, und die ist unzu- 
verlässig und darf das auch sein. Entsprechend unterschiedlich fallen die beiden Live-Aufnahmen aus, die von 106a verfügbar sind (Solo [Brussels] 1985; 19 [Solo] Compositions, 1988). Eine sozusagen textgetreue, die einzelnen Punkte der Komposition der Reihe nach berücksichtigende Umsetzung liegt uns von Matthew Welch vor, der $106 a$ im Studio akkurat nach der Vorlage eingespielt hat - auf dem Dudelsack (10 [Solo Bagpipe] Compositions 2000). Doch wissen wir dadurch wirklich, wie sich $106 a$ korrekt gespielt anhört? In formaler Hinsicht schon, aber nicht dem musikalischen Gehalt nach. Eine musikalisch präzise Version von $106 a$ kann es gar nicht geben, da für »Long Sound >Winnie« « und dergleichen keine genauen akustischen Modelle existieren, die als klangliches Richtmaß heranzuziehen wären.

Diese Uneindeutigkeit ist von Braxton beabsichtigt. Wer sich als Interpret auf die Solostücke einlässt, ist aufgefordert, individuelle Lösungen zu finden, wie die Worte und Zeichen in Musik zu übersetzen sind. Die mitunter mehr als skurrilen Aufforderungen verlangen vom Instrumentalisten in jedem Fall Witz und Phantasie, aber nicht unbedingt eine brillante Spieltechnik: »Dog barks« (Composition No. 99g), »Japanese steadyness« (No. 99e), »Squeak« (No. 106f) oder, in der »Bach piece« benannten Composition No. 118c: » so back to VIENNA (smile)«, um nur einige Beispiele zu nennen.

Das vom Instrumentalisten Braxton kontrolliert erweiterte Vokabular, von dem die Rede war, erstreckt sich auch auf den Einsatz von Geräuschen (Klappengeräuschen, Atemgeräuschen usw.) sowie auf bewusst inakkurate Artikulation und »false sounds«. Das Ergebnis hört sich dann streckenweise so unakademisch an wie ein Objekt von Joseph Beuys aussieht, und dieser ungewöhnliche Vergleich ist gar nicht mal so weit hergeholt. Die Phil Woods dieser Welt mögen das in ihrer Engstirnigkeit mit fehlender Expertise verwechseln, doch damit hat es nichts zu tun.

Ein weiteres Feld, auf dem Braxton mit Virtuositätskonventionen bricht, sind Jazzstandards. Seine zahlreichen Einspielungen von Fremdkompositionen sind alles, nur keine schulmäßigen Interpretationen der Originale. »Ich spiele die Titel seit über 40 Jahren, da ist es mir ein Bedürfnis, sie lebendig zu erhalten, indem ich vom Üblichen abweiche «, hat Braxton einmal in einem Interview seinen Ansatz erläutert (Hoyer 2006: 57). Buchstäblich alle Verwendungsweisen und Umstrukturierungen der Standard-Grammatik scheinen erlaubt, nur Einfallslosigkeit, Imitation und Langeweile nicht. Die Ergebnisse - jüngst eine 7-CD-Box mit Kompositionen von Lennie Tristano und den Musikern aus dessen Umfeld (Quintet [Tristano] 2014) - können Jazzpuristen schon mal zur Weißglut bringen, zählen aber ohne Wenn und 
Aber zu den atemberaubenden Lesarten des Jazzkanons (vgl. z.B. Wilson 1993: 209ff.; Hawkins 2016).

Wie wenig Braxton an der Erfüllung oder Überbietung von Virtuositätsmaßstäben liegt, unterstreicht er auf eigentümliche Weise seit 1994, als er das erste Mal mit Auftritten überraschte, in denen er in einem Quartett als Pianist zu hören war. Zig Auftritte und Aufnahmen - darunter auch die Tristano-Box - in unterschiedlichen Formationen und als Solopianist sollten folgen; ausnahmslos stehen Standards auf dem Programm. Braxton hatte in den Jahren vor seinem ersten Auftritt als Pianist eifrig Klavierspielen geübt, aber reichte das, und wenn ja, wofür?

Man wird ihn nicht als Dilettanten am Piano bezeichnen können, dafür ist sein Spiel zu versiert, planvoll und durchdacht, kurz: zu gekonnt (vgl. Day 1998). Insofern fraglos kompetent und kreativ. Also, nach obiger Definition, regelrecht virtuos? Nein, so kompetent nun auch wieder nicht. Man erkennt nach einer Weile, dass sein Gestaltungsrepertoire als Pianist einigermaßen beschränkt ist (vergleichbar in der Malerei mit dem wunderbaren Henri Rousseau oder im Jazz mit dem Violinspiel Ornette Colemans). Und man ahnt, dass er sich diese Begrenzung des Vokabulars nicht mutwillig auferlegt hat, wie es manche bildendenden Künstler in bestimmten Werkphasen tun. Wenn man Braxton als Klaviervirtuosen bezeichnen würde (oder Coleman als virtuosen Violinisten, Rousseau als virtuosen Maler), wie wollte man dann die ungleich größere Meisterschaft, Ausdruckspalette und Instrumentalbeherrschung eines Cecil Taylor, Keith Jarrett oder einer Irène Schweizer bezeichnen? Deren Möglichkeiten sind selbstredend auch nicht unbegrenzt, scheinen aber so. Beim Pianisten Braxton sind die spieltechnischen Limitationen offensichtlich, er will sie auch gar nicht überspielen oder verheimlichen, er reizt seine begrenzten Möglichkeiten vielmehr so geschickt und variantenreich aus wie ein Haiku-Dichter die Begrenzung der lyrischen Form. Und während man dem Piano-Novizen dabei zuhört, wie er die Jazzstandards aus den Angeln hebt, wird einem nebenbei klar, dass Meisterschaft und Instrumentalbeherrschung nur Mittel zum Zweck sind und dass Virtuosität in der Kunst wahrlich nicht der Zweck an sich ist.

"If the music is played too correctly it was propably played wrong « (Braxton 1989, o.S.), leitet Braxton sein Werkverzeichnis ein. Damit will er selbstverständlich nicht zur Stümperei oder Nachlässigkeit ermutigen. Das Bonmot ist Ausdruck seiner non-perfektionistischen Grundeinstellung. Wenn alles exakt und vollkommen ist, dann gibt es keine Entwicklung, keine Bewegung, keine Kreativität. Deshalb bevorzugt Braxton auf hohem Niveau das Unvollkommene und Inkorrekte. Als Komponist und Bandleader hat er es darauf abgesehen, beides zu provozieren. Konkret geschieht dies durch Über- 
forderung. Braxton führt sich - nicht nur als Pianist - und seine Musiker/ innen (von seinem Publikum ganz zu schweigen) unentwegt an die Grenzen des Machbaren und häufig ein wenig darüber hinaus, was bei den exquisiten Musiker/innen, mit denen er sich umgibt, gar nicht einfach ist: Stücke gespielt in irrwitzigem Tempo, andere, die Mikroimprovisationen von wenigen Sekunden und den permanenten Wechsel zwischen unterschiedlichen Kompositionen verlangen, Aufführungen, die ohne Unterbrechung acht Stunden dauern (Sonic Genome), Kompositionen, in denen die Musiker/innen ihre Instrumente spielen, iPods bedienen, graphische Notationen entziffern, Spielanweisungen geben und welche empfangen - und das alles zur gleichen Zeit (Echo Echo Mirror House), vertrackte Opernlibretti, die aus ellenlanger philosophischer Prosa bestehen (Trillium) und anderes mehr. Jeder noch so versierte Musiker wird unter solchen Anforderungen aus seiner Komfortzone gescheucht. Das ist ein Grund, weshalb in Braxtons Werk von lächerlicher Virtuosität so wenig zu hören ist: Jenseits der Komfortzone sind die Gelegenheiten rar, Kabinettstücke routiniert abzuspulen.

Nun weiß jeder, der Braxtons Musik ein wenig kennt, dass vor allem in seinen Quartetten der 1970er, 1980er und frühen 1990er Jahre ausgiebig und glanzvoll improvisiert wurde. Doch selbst in seiner verhältnismäßig jazznahen Quartett-Musik hat Braxton den Spielplatz virtuoser Gefälligkeit zusehends eingeschränkt, namentlich mit der Entwicklung seiner Co-ordinate Music. Das ist ein ausgetüfteltes Collage-System bestehend aus heterogenen Eigenkompositionen und Improvisationen (vgl. Wilson 1993: 112ff.; Heffley 1996: 336ff.). Der Korpus der Co-ordinate Music wechselt während einer ungefähr 60-minütigen Aufführung wie ein Kaleidoskop fortwährend die Gestalt, durch die sich die Musikerinnen und Musiker hindurchschleusen, während sie sie formen. Bei der »navigation through form«, wie Braxton dieses Gestaltungsprinzip nennt (vgl. Corbett 1994), steht das kollektive Modulieren eines Klanggebildes im Vordergrund. Der klassische Jazzsolist bekommt darin noch hin und wieder eine Bühne, doch das virtuose Solo ist lediglich eine unter vielen Facetten im Gesamtbild und nicht dessen Fluchtoder Höhepunkt.

\subsection{Virtuose Ensembles}

An der Entwicklung von Braxtons Quartett-Musik lässt sich bereits ein Motiv ablesen, das besonders in der zweiten Hälfte seiner Karriere bestimmend werden sollte. Diese beginnt nach der Auflösung seines Quartetts 1993 mit einem neuen Kompositionsmodell, das den Namen Ghost Trance Music (GTM) trägt. In der GTM ebenso wie in allen darauf folgenden Komposi- 
tionsmodellen Braxtons (vgl. Wooley 2016a), und nicht zuletzt in seinen Orchesterwerken und seinem ausladenden Opernzyklus Trillium (dem Magnum Opus seines Spätwerks), macht sich Virtuosität weniger an einzelnen Glanzleistungen fest; die Spielräume hierfür werden durchweg minimiert. Maximiert - oder besser: demokratisiert - werden stattdessen die Möglichkeiten der kollektiven Formgebung und die Gestaltungsoptionen der Musiker und Musikerinnen. Mit anderen Worten: Virtuosität verlagert sich von der kreativen Einzelperson auf das kreative, demokratisierte Ensemble.

Wie solch ein demokratisches Ensemble funktioniert, ist an besagter GTM zu studieren (vgl. Dicker 2016). Die GTM setzt sich aus drei Materialsorten zusammen. Erstens aus einer ausnotierten, bis zu 80 Seiten umfassenden Basiskomposition, der »Primary Melody«.

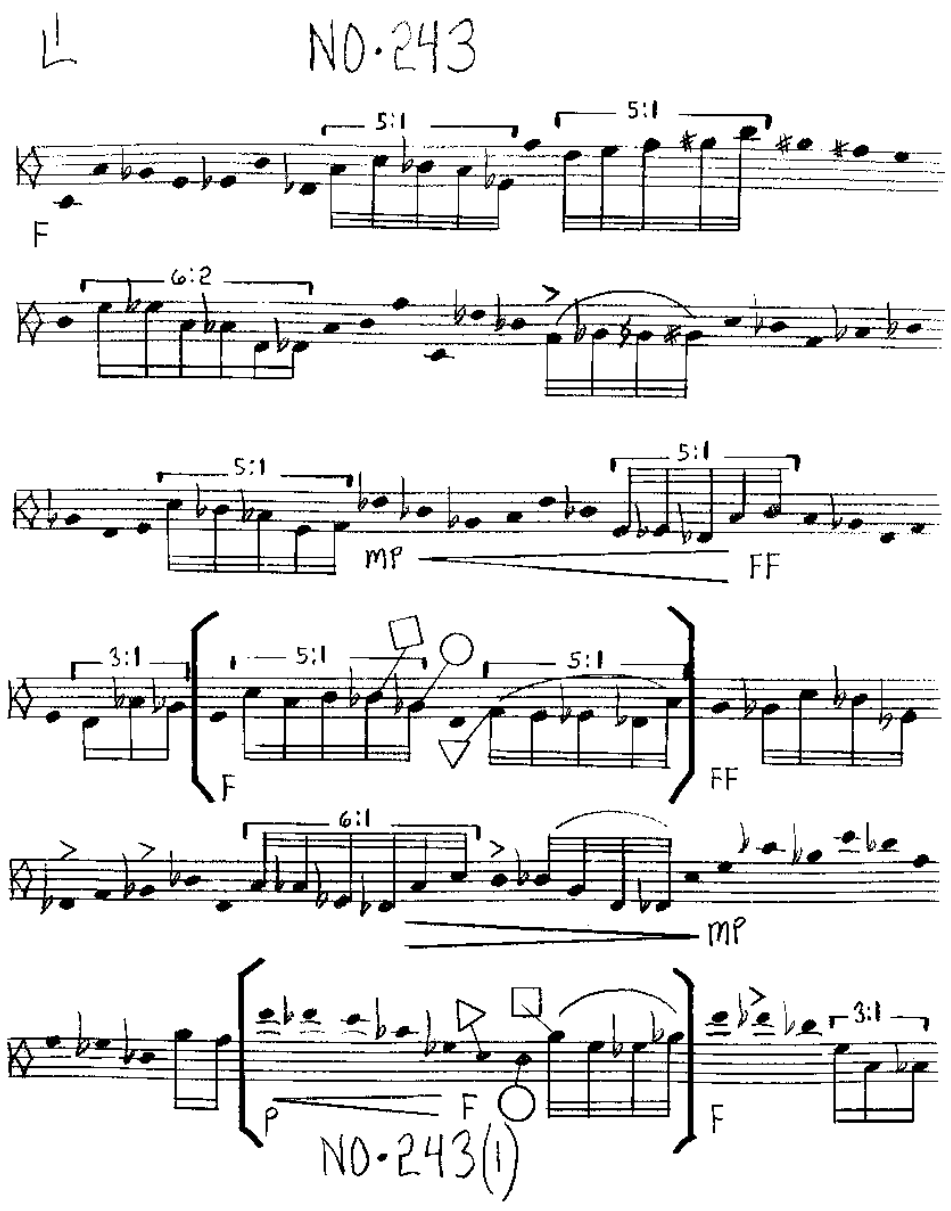

(c) Anthony Braxton / Tri-Centric Foundation

Abbildung 1: Ghost Trance Music. Anthony Braxtons Composition No. 243, erste Partiturseite

Sie stellt innerhalb der Infrastruktur einer Gesamtkomposition gewissermaßen die Hauptstraße dar, auf der sich das Ensemble fortbewegt. Daneben 
gibt es viele Abzweigungen und Parallelstraßen, die mal von diesem, mal von jenem Teil des Ensembles genutzt werden. Die beiden anderen Materialsorten legen den groben Verlauf dieser zusätzlichen Wege fest. Das »Secondary Material « umfasst eine Reihe von einseitigen Partituren - wie alles hier aus Braxtons Feder -, die als Solo, im Duo, Trio oder anderen Kleinformaten zu spielen sind.
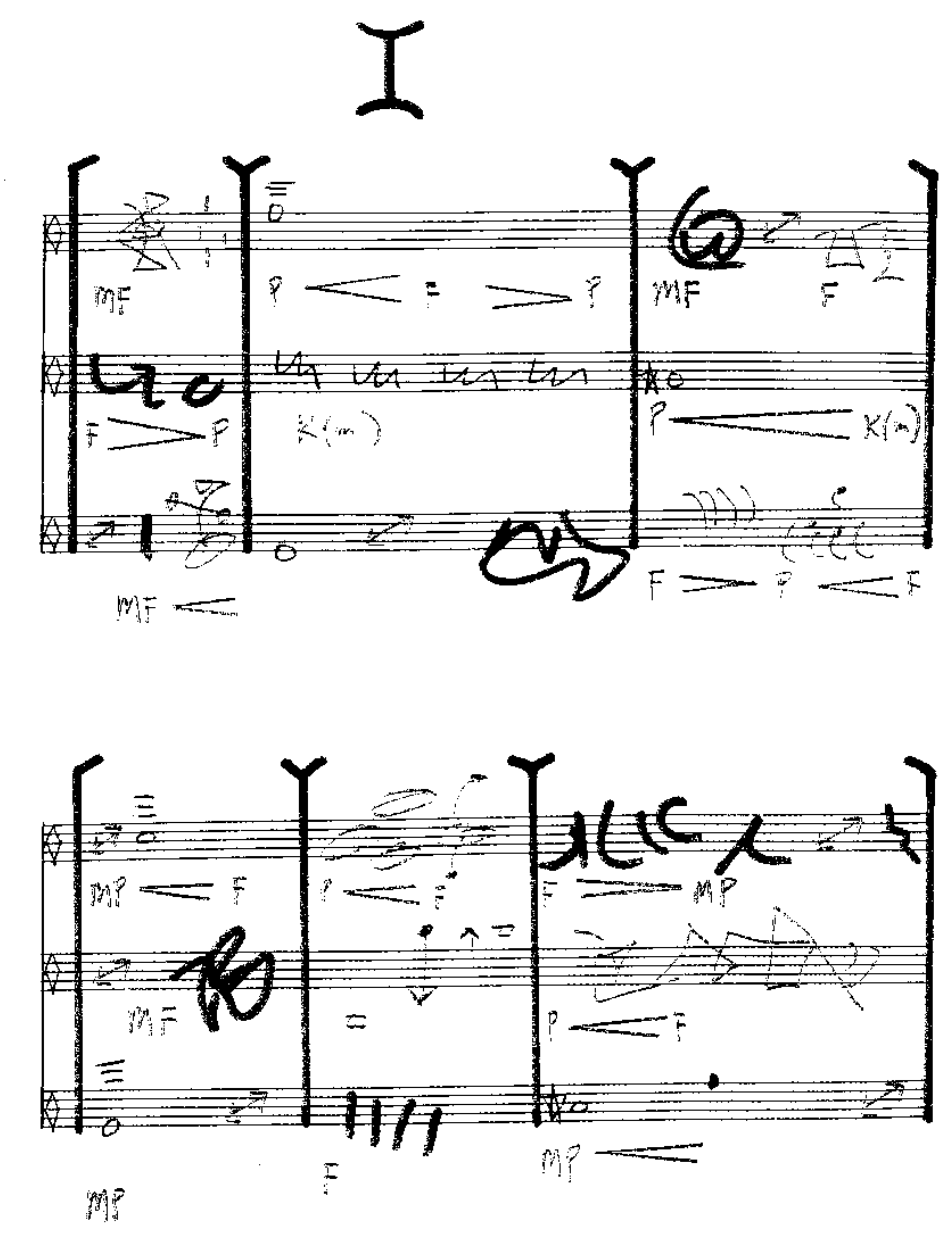

(C) Anthony Braxton / Tri-Centric Foundation

Abbildung 2: Anthony Braxtons Composition No. 243. Secondary Material. Nr. I

Die Zeitpunkte, an denen das im Laufe einer meist rund einstündigen, ununterbrochenen Aufführung geschehen kann, werden über Dreiecke innerhalb der Basiskomposition angezeigt. Die Ensemblemusiker/innen entscheiden eigenständig, zu welchem »Secondary Material« sie greifen, und sie wählen gegebenenfalls Mitspieler/innen aus. Die Verständigung kann im Vorfeld des Konzerts geschehen oder, was vorwiegend der Fall ist, während der Aufführung spontan über ein Handzeichensystem. Tauchen in der Partitur der »Primary Melody« Quadrate auf, dann dürfen die Musiker/innen sich in der- 
selben Weise darüber verständigen, welches »Tertiary Material« sie einbeziehen wollen. Unter diesen dritten Materialtyp fallen praktisch sämtliche Kompositionen Braxtons (um die 500), aus denen vor dem Konzert einige Musiker/innen, die Sectionleaders, eine Auswahl treffen. Insgesamt ein Haufen Noten also! Und wo ist bei all dem Platz für Improvisation? Den symbolisieren Kreise innerhalb der Basiskomposition. Die Musiker/innen haben dann die Wahl, nach Lust und Laune zu improvisieren, oder sich dabei, allein oder mit anderen, auf einen Typus der Language Music zu beziehen. Es steht ihnen aber auch frei, weiter der »Primary Melody« zu folgen oder sich auf eine nächste Gruppenaktivität vorzubereiten. Einen Bandleader gibt es auch, er ist dafür verantwortlich, dass alle Mitglieder des Ensembles ab und an auf der Hauptstraße zusammenfinden. - Als Hörbeispiel sei die grandiose CD/DVD-Box 9 Compositions (Iridium) 2006 empfohlen.

Die Virtuosität solch eines Ensembles ergibt sich, wie man sieht, nicht aus der Summe virtuoser Solisten, die sich innerhalb eines vorgegebenen Schemas reihum profilieren, wie man es aus dem traditionellen Big Band Jazz kennt. Virtuos ist das Ensemble in der gemeinsamen Gestaltung eines musikalischen Gebildes. Das erinnert an die Gruppenimprovisationen der ersten und zweiten Jazzavantgarde und an europäische Improv-Projekte, an denen sich Braxton auch wiederholt beteiligt hat. Die musikalische Formgebung geschieht in Braxtons Konzepten jedoch nicht vorrangig über offene oder gelenkte Improvisationen, sondern folgt einer festgelegten Grammatik, deren Bestandteile, Strukturen und Regeln die Ensemblemitglieder erlernen müssen, um sie kompetent und kreativ nutzen zu können. Dieser distinktiven Grammatik ist es zu verdanken, dass sämtliche Aufführungen der GTM ungeachtet aller einkalkulierten Unvorhersehbarkeit und der strukturellen Offenheit eine unverwechselbare Textur besitzen: die Handschrift des Komponisten. Das ist ein Charakteristikum aller Kompositionsmodelle, die Braxton entworfen hat.

\subsection{Virtuoses Komponieren}

Die GTM-Partituren sind eine Augenweide: klar wie mathematische Formeln und rätselhaft wie eine Geheimlehre. Von Karlheinz Stockhausen hat Braxton gelernt, den Niederschriften seiner Kompositionen eine ästhetische Qualität zu geben, die über die reine Funktionalität der Notenschrift hinausweist. Mittlerweile hängen seine Partituren in Galerien und Ausstellungen (z.B. Kramer 2015). Als für sich stehende Kunstwerke gehen besonders seine visuellen Notationen und seine gezeichneten Kompositionstitel durch (vgl. Lock 2008). Einen abermals herausgehobenen Stellenwert hat eine Gruppe 
graphischer, nichtlinearer »Partituren«, die in der Anfang 2000 entwickelten Falling River Music und in der Diamond Curtain Wall Music zum Einsatz kommen - zwei konzeptionelle Prototypen, mit denen Braxton das Feld intuitiver Improvisation auskundschaftet (vgl. Hoyer 2006: 58).

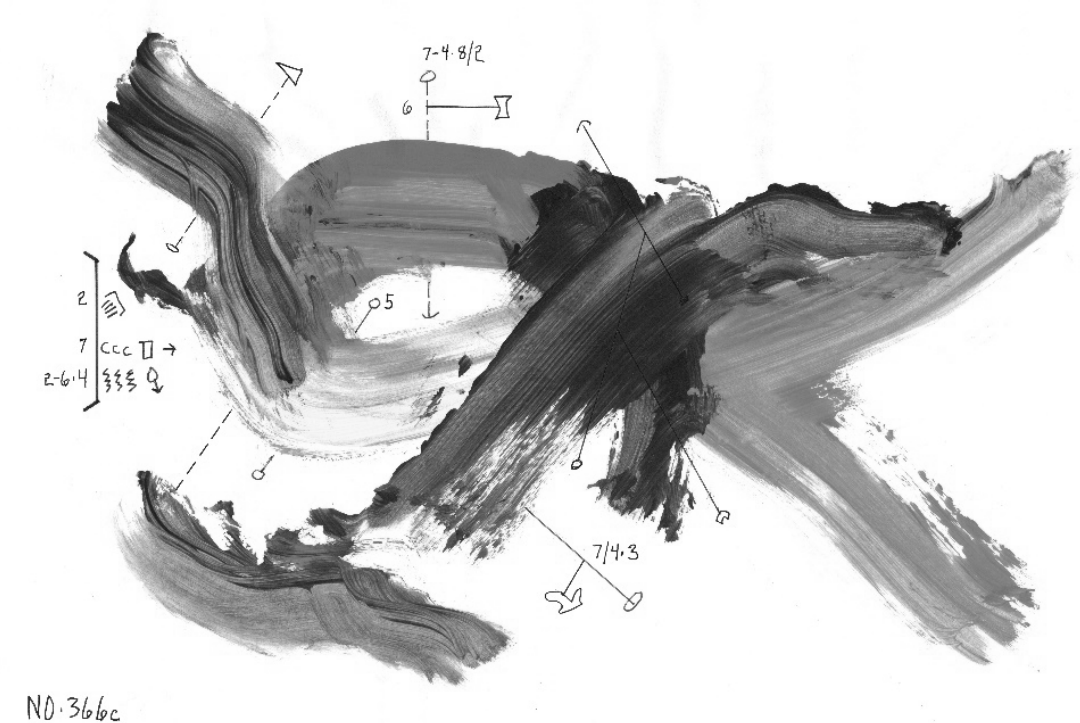

(C) Anthony Braxton / Tri-Centric Foundation

Abbildung 3: Falling River Music. Anthony Braxtons Composition No. 366c

Sollten rotierende Pinselstriche, farbige Linien, Punkte, Zahlen- und Zeichenkolonnen eine sinnvolle Spielvorgabe abgeben, wird sich manch einer beim Betrachten dieser notenschriftlosen Leadsheets denken, dann wären doch die Museen der Moderne voll von unentdeckten »Partituren«. Ja, aber warum eigentlich nicht? Wenn Partituren nicht mehr unbedingt der musikalischen Realisation bedürfen, um als ästhetische Objekte wahrgenommen zu werden, warum sollten dann Werke der Bildenden Kunst nicht als Partituren heranzuziehen sein? Braxton untermauert mit seinen visuellen Kompositionen, dass die Korrespondenzen zwischen (frei) improvisierter Musik und (abstrakter) Malerei (vgl. Wilson 1999: 68ff.) noch längst nicht ausgereizt sind.

Als Komponist ist er selbstredend nicht nur kreativ in der ästhetischen Gestaltung differenter Spielvorgaben, sondern allen voran in der Weiterentwicklung von musikalischen Vokabularen und Grammatiken. »I've never really been interested in anarchy«, hat er sich 1978 von der brachialen Free Jazz-Attitüde abgegrenzt, »but rather l've been interested in the initiation of alternative languages as a means to reconnect to what I call the metareality implications of world culture« (Enstice / Rubin 1994: 51). So vorzüg- 
lich und originell Braxton seit einem halben Jahrhundert die Sprache der transidiomatischen Musik bereichert, ist er ein beispielhafter Protagonist virtuosen Komponierens, der Maßstäbe setzt.

Er geht dabei, wie schon deutlich geworden sein dürfte, systematisch vor, wobei sein System nicht in Marmor gemeißelt, sondern dynamisch und entwicklungsoffen ist (vgl. Hoyer 2014). Sein Gesamtkonzept, hat Braxton kürzlich erklärt, »is a system of becoming, not a system of arriving « (Mollesen 2016).

Die Grundkomponente dieses Systems besteht in der kompositorischen Arbeit mit drei Notationskategorien. Braxton unterscheidet »stable logics« (z.B. das abendländische Notensystem), »mutable logics« (offen gehaltene Spiel- und Improvisationsaufforderungen, z.B. die Language Music) und »synthesis logics« (synergetische Notationsverfahren) (vgl. Heffley 2000).

Auf diese Typologie greift der Komponist Braxton zurück, um in ein ums andere Mal verblüffenden Varianten das Geplante und das Spontane, das Wiederholbare und das Einmalige, das Vereinbarte und das Unvorhergesehene miteinander zu verschränken. Sein Gesamtwerk ist eine einzige ideenreiche, um nicht zu sagen virtuose Antwort auf die Frage, wie aus diesen drei »Logiken« unerhörte Musik entstehen kann.

\section{Literatur}

Bailey, Derek (1987). Improvisation. Kunst ohne Werk. Hofheim: Wolke.

Böttcher, Jan (2011). Das Lied vom Tun und Lassen. Reinbek bei Hamburg: Rowohlt.

Braxton, Anthony (1985). Tri-Axium Writings 1-3. Synthesis Music (New Hampshire: Frog Peak Music).

Braxton, Anthony (1988). Composition Notes. Book E. Synthesis Music (New Hampshire: Frog Peak Music).

Braxton, Anthony (1989). Catalog of Works. Synthesis Publishing (New Hampshire: Frog Peak Music).

Corbett, John (1994). »Anthony Braxton. From Planet to Planet.«In: Ders., Extended Play. Sounding Off from John Cage to Dr. Funkenstein. Durham, London: Duke University Press, S. 209-217.

Day, Steve (1998). »Jelly Roll Braxton. Listening to Anthony Braxton as a Piano Player.« In: Ders., Two Full Ears. Listening to Improvised Music. Chelmsford: Soundworld Publ., S. 87-95.

Dicker, Erica (2016). »Ghost Trance Music.«In: Sound American 16: The Anthony Braxton Issue, http://soundamerican.org/sa_archive/sa16/sa16-ghost-trancemusic.html (Stand vom 21.3.2017).

Enstice, Wayne / Rubin, Paul (Hg.) (1994). Jazz Spoken Here: Conversations with 22 Musicians. New York: Da Capo Press.

Feige, Daniel Martin (2014). Philosophie des Jazz. Berlin: Suhrkamp. 
Hawkins, Alexander (2016). »Anthony Braxton - Quintet (Tristano) 2014.« In: LondonJazzNews vom 24. April, http://www.londonjazznews.com/2016/04/reviewessay-2-of-3-anthony-braxton.html (Stand vom 21.3.2017).

Heffley, Mike (1996). The Music of Anthony Braxton. New York: Excelsior Music Publishing Company.

Heffley, Mike (2000). Braxton's System: An Artificer's Intelligence, https://www. academia.edu/2320225/Braxtons_System_An_Artificers_Intelligence (Stand vom 21.3.2017).

Hoyer, Timo (2005). Tugend und Erziehung. Zur Grundlegung der Moralpädagogik in der Antike. Bad Heilbrunn: Klinkhardt.

Hoyer, Timo (2006). »Musik fürs 21. Jahrhundert: Anthony Braxton. «In: Jazzthetik, H. 3, S. 54-58.

Hoyer, Timo (2014). »Notorisch im Fluss. Anthony Braxtons unbändige Produktivität. «In: Neue Zeitschrift für Musik 175, Nr. 4, S. 54-55.

Hoyer, Timo (2017). »Pop, Pop, Antipop. «In: Was ist Popmusik? Hg. v. Timo Hoyer, Carsten Kries und Dirk Stederoth. Darmstadt: Wissenschaftliche Buchgesellschaft (Druck in Vorbereitung).

Jost, Ekkehard (1982). Sozialgeschichte des Jazz in den USA. Frankfurt/M.: Fischer.

Keller, Gottfried (1919). Der grüne Heinrich. Nach der ersten Fassung 1854-55. Berlin: Brandusche Verlagsbuchhandlung.

Kramer, Michael (Hg.) (2015). The Freedom Principle. Experiments in Art and Music. 1965 to Now. Chicago: University of Chicago Press.

Lemke-Matwey, Christine (2016). »Jugendstil mit Schwimmflossen.«In: Die Zeit 71, Nr. 25, S. 49.

Lewis, George E. (2008). A Power Stronger Than Itself. The AACM and American Experimental Music. Chicago: University of Chicago Press.

Litweiler, John (1988). Das Prinzip Freiheit. Jazz nach 1958. Schaftlach: Oreos.

Lock, Graham (1995). „A Vision of Forward Motion. Notes on the Evolution of Anthony Braxton's Solo Music. In: Mixtery. A Festschrift for Anthony Braxton. Hg. v. Graham Lock. Devon: Stride Publications, S. 48-54.

Lock, Graham (2008). »)What I Call a Sound and Notations for Improvisers. «In: Critical Studies in Improvisation 4, Nr. 1, http://www.criticalimprov.com/article/view/462/992 (Stand vom 21.3.2017).

Márquez, Gabriel García (1997). Hundert Jahre Einsamkeit. Köln: Kiepenhauer \& Witsch (33. Aufl.).

Mollesen, Kate (2016). »Anthony Braxton still Knows no Boundaries. «In: The Herald vom 2. November, http://www.heraldscotland.com/arts_ents/14836989. Antho ny_Braxton_still_knows_no_boundaries (Stand vom 21.3.2017).

Noglik, Bert (1981). Jazzwerkstatt international. Berlin: Verlag Neue Musik.

Radano, Ronald M. (1993). New Musical Figurations. Anthony Braxton's Cultural Critique. Chicago, London: University of Chicago Press.

Wilmer, Valerie (1992). As Serious As Your Life. John Coltrane and Beyond. London: Serpent's Tail.

Wilson, Peter Niklas (1993). Anthony Braxton. Sein Leben, seine Musik, seine Schallplatten. Waarkirchen: Oreos.

Wilson, Peter Niklas (1999). Hear and Now. Gedanken zur improvisierten Musik. Hofheim: Wolke.

Wooley, Nate (Hg.) (2016a). Sound American 16: The Anthony Braxton Issue, http://soundamerican.org/sa_archive/index.html (Stand vom 21.3.2017). 
Wooley, Nate (2016b). »Anthony Braxton's Language Music. In: Sound American 16: The Anthony Braxton Issue, http://soundamerican.org/sa_archive/sa16/sa16language-music.html (Stand vom 21.3.2017).

\title{
Diskographie
}

Braxton, Anthony (o.J.). Solo (Brussels) 1985. Braxton Bootleg BL009, http:// tricentricfoundation.org/all-releases/bootleg (Stand vom 21.3.2017).

Braxton, Anthony (1989). 19 (Solo) Compositions, 1988. New Albions Rec. NA 023 CD.

Braxton, Anthony (2000). 10 [Solo Bagpipe] Compositions 2000. Performed by Matthew Welch. Parallactic 36.

Braxton, Anthony (2007). 9 Compositions (Iridium) 2006. Firehouse 12 Rec. FH1204-03-001 (9 CDs, 1 DVD).

Braxton, Anthony (o.J.). Quintet (Tristano) 2014. New Braxton House NBH 905 (7 CDs).

\begin{abstract}
Based on an explanation of the meaning of virtuosity and a brief comment on virtuosity in traditional jazz, the contribution reconstructs four variations of virtuosity in the work of the jazz avant-gardist Anthony Braxton: autonomous virtuosity, undermined virtuosity, the virtuosic ensemble, and the virtuosic composer.
\end{abstract}

